\title{
HD 11397 and HD 14282: two new barium stars? ${ }^{\star \star \star \star}$
}

\author{
L. Pompéia ${ }^{1}$ and D. M. Allen ${ }^{2}$ \\ 1 IP\&D, Universidade do Vale do Paraíba, Av. Shishima Hifumi, 2911, São, José dos Campos, 12244-000 SP, Brazil \\ e-mail: pompeia@univap.br \\ 2 Centre for Astrophysics Research, STRI and School of Physics, Astronomy and Mathematics, University of Hertfordshire, \\ Hatfield, UK e-mail: d.moreira-allen@herts.ac.uk
}

Received 3 March 2008 / Accepted 27 June 2008

ABSTRACT

\begin{abstract}
Context. We have performed a detailed abundance analysis of the content of s-process elements of two dwarf stars with suspected overabundace of those elements. Such stars belong to a special kinematic sample of the solar neighborhood, with peculiar kinematics and different chemical abundances when compared to "normal" disk stars.

Aims. We aim to define if those stars can be identified as barium stars, based on their s-process elements abundances, and their classification, i.e., if they share their chemical profile with strong or mild barium stars. We also intend to shed light on the possible origins of the different kinds of barium stars.

Methods. Spectra have been taken by using the FEROS spectrograph at the $1.52 \mathrm{~m}$ telescope of ESO, La Silla. Abundances have been derived for 18 elements, by matching the synthetic profile with the observed spectrum.

Results. We have found that HD 11397 shows a mild enhancement for most of the $s$-process elements as well as for some $r$-process elements. This star seems to share its abundance profile with the mild Ba-stars. Although showing some slight chemical anomalies for $\mathrm{Y}, \mathrm{Sr}, \mathrm{Mo}$, and $\mathrm{Pb}, \mathrm{HD} 14282$ depicts a chemical pattern similar to the normal stars with slight $s$-process enhancements.
\end{abstract}

Key words. stars: abundances - stars: AGB and post-AGB - stars: chemically peculiar

\section{Introduction}

Some fields in astrophysics are particularly complex due to the interconnection of many different and, at the same time, important factors. Barium stars certainly belong to one of those fields. These are chemically peculiar stars with enhancements of $s$-process elements and carbon, with luminosities below the threshold for the onset of thermal pulses in the AGB (Asymptotic Giant Branch) phase. They were identified for the first time by Bidelman \& Keenan (1951) as peculiar red giants, and since then many studies have been performed in order to better understand their nature and origin (e.g. McClure 1984; Lambert 1988; Luck \& Bond 1991; Lambert et al. 1993; North et al. 1994; Jorissen et al. 1998; Liang et al. 2000). s-process elements are produced along the thermal instability phase of the AGB (TP-AGB) (see e.g. Iben 1991) at the interpulse period triggered by the ${ }^{13} \mathrm{C}(\alpha, n){ }^{16} \mathrm{O}$ reaction, and marginally during the convective thermal pulses by the ${ }^{22} \mathrm{Ne}(\alpha, n)^{25} \mathrm{Mg}$ reaction (e.g. Wallerstein et al. 1997; Busso et al. 1999). The $s$-process nucleosynthesis takes place slightly above the He-rich, C-rich shell, and during thermal pulses, material enriched with $s$-process and carbon ashes is mixed to the H-rich envelope by the third dredge-up. As a consequence of this process, the atmospheric abundances of TP-AGB stars slowly change with time, with increasing $s$ and $\mathrm{C}$ content, eventually forming a carbon star.

\footnotetext{
* Based on spectroscopic observations collected at the European Southern Observatory (ESO), within the Observatório Nacional $\mathrm{ON} / \mathrm{ESO}$ and ON/IAG agreements, under FAPESP project No. 1998/10138-8.

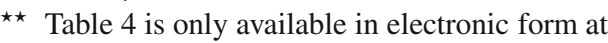

http://www . aanda.org
}

As Ba stars are in an evolutionary stage before the AGB, their surface abundances are puzzling. Two hypotheses have been suggested to explain their chemical peculiarity. The first and most accepted is the mass-transfer scenario within a binary system by an AGB star to an unevolved (less massive) companion (McClure et al. 1980). Along the AGB, stars develop strong stellar winds, with mass-loss rates, $\dot{M}$, from $10^{-7}$ to $10^{-5} M_{\odot} \mathrm{yr}^{-1}$, contaminating the environment with their material (Busso et al. 2001). Therefore binary companions near TP-AGB stars are expected to inherit large amounts of this material and change their atmospheric carbon and $s$-process elements abundances. In the second hypothesis, Ba stars are formed from ISM clouds previously contaminated by AGB activity (e.g. Beveridge \& Sneden 1994). Studies about the binary incidence in $\mathrm{Ba}$ stars have been extensively performed and have strengthened the first hypothesis (McClure 1984; McClure \& Woodsworth 1990; Jorissen \& Mayor 1988; North \& Duquennoy 1992; Karakas et al. 2000). Jorissen et al. (1998) for example studied the radial velocity of a sample of Ba stars, with mild or strong peculiarities. They have found that 35/37 of the strong and 34/40 of the mild Ba stars are binaries, "compatible with the hypothesis that all the observed stars are binary systems". Those results strongly support the binary origin for the Ba stars (see Pols et al. 2003; and Jorissen \& Van Eck 2005; for a detailed discussion), although special cases might exist such as the chemically peculiar stars from $\omega$ Cen (Norris \& Da Costa 1995; Smith et al. 2000).

McClure et al. (1980) and Sneden et al. (1981), based on the work by Warner (1965), divided the Ba stars according to their chemical anomalies in strong and mild Ba stars. The classification index takes into account the Ba II $4554 \AA$ line strength, and ranges from 1 to 5 . Strong Ba stars are more contaminated by 
Table 1. Photometric and spectroscopic parameters for HD 11397 and HD 14282.

\begin{tabular}{ccccccc}
\hline \hline Name & $\log g_{\text {Spec }}$ & $\log g_{\text {Hip }}$ & $T_{\text {Spec }}$ & {$[\mathrm{Fe} / \mathrm{H}]_{\text {Spec }}$} & {$[\mathrm{Fe} / \mathrm{H}]_{\mathrm{Gen}}$} & $\xi\left(\mathrm{km} \mathrm{s}^{-1}\right)$ \\
\hline HD 11397 & $4.15 \pm 0.2$ & 4.34 & $5400 \pm 100$ & $-0.72 \pm 0.15$ & -0.78 & $0.80 \pm 0.15$ \\
HD 14282 & $3.70 \pm 0.2$ & 3.91 & $5800 \pm 100$ & $-0.60 \pm 0.15$ & -0.59 & $1.00 \pm 0.15$ \\
\hline
\end{tabular}

$s$-process elements and carbon and have an index of $\sim 3$ to 5 ; for the mild $\mathrm{Ba}$ stars the index is $\$ 2$. The process (or processes) by which a normal star becomes a strong or a mild Ba star have been discussed by some authors but is still lacking a definite conclusion (e.g. Pols et al. 2003; Liang et al. 2003; Jorissen \& Van Eck 2005; Smiljanic et al. 2007). The orbital period of the binary system, the $s$-process efficiency during the AGB phase of the more massive star, the type and efficiency of the mass-transfer mechanism, the metallicity of the stars, and the mass of the future Ba star which affects the dilution degree of the transferred material, may all play a role in the final abundances. Jorissen et al. (1998) have found that Ba stars with very similar orbital periods show different $s$-enhancements, indicating that other parameters are involved. They suggest that mild, strong and Pop.II CH stars (which show very large $s$-enhancements) correspond to a sequence of increasingly older and more metal-deficient populations. Smiljanic et al. (2007) claim that mild and strong Ba stars show similar iron content, and their difference is probably connected to the neutron exposure during the $s$-process operation. Higher neutron exposures would produce strong Ba stars, while mild $\mathrm{Ba}$ stars have accreted matterial exposed to weaker neutron exposures.

In the present work we report chemical abundances for two stars from the bulgelike sample of Michel Grenon (Grenon 1999, 2000), HD 11397 and HD 14282. Those stars belong to a kinematically selected sample of the solar neighborhood, the bulgelike stars (Pompéia et al. 2002, 2003). The bulgelike stars have very eccentric orbits, with eccentricities e $>0.25$ and small pericentric distances with $R_{\mathrm{p}} \leq 3.5 \mathrm{kpc}$. Therefore they were probably born near the galactic bulge (Pompéia et al. 2003, 2008). The chemical abundances of the bulgelike stars have been previously studied (Pompéia et al. 2003) and their Ba, Zr, La and Y content have been determined. HD 11397 and HD 14282 have shown abundance anomalies in their $s$-process content, with enhanced $[\mathrm{Ba} / \mathrm{Fe}]$ and $[\mathrm{La} / \mathrm{Fe}]$ ratios when compared to the other bulgelike stars (see Fig. 8 of Pompéia et al. 2003). HD 14282 also shows overabundant $[\mathrm{Zr} / \mathrm{Fe}]$ ratios when compared to the other bulgelike stars. In order to properly classify the present stars and to perform a full analysis of their neutron-capture elements profile, we have inferred their abundances for $\mathrm{C}, \mathrm{N}, \mathrm{La}, \mathrm{Ba}, \mathrm{Nd}, \mathrm{Sm}, \mathrm{Sr}$, $\mathrm{Zr}, \mathrm{Y}, \mathrm{Mo}, \mathrm{Ru}, \mathrm{Ce}, \mathrm{Pr}, \mathrm{Gd}, \mathrm{Dy}, \mathrm{Hf}$ and $\mathrm{Pb}$, and for the $r$-process element Eu.

This work is divided as follows: in Sect. 2 we describe the observations and reduction procedures, the stellar parameters determination and the model atmospheres are described in Sect. 3, the abundance calculations and their results are given in Sect. 4, in Sect. 5 we discuss our results, and in Sect. 6 we give a summary of the paper.

\section{Observations and stellar parameters}

Sample stars have been observed at the $1.52 \mathrm{~m}$ telescope of ESO (European Southern Observatory), La Silla, in September 1999. The spectra were obtained using the FEROS spectrograph (Fiber-fed Extended Range Optical Spectrograph) with wavelength range 356 to $920 \mathrm{~nm}$ and a resolution of $R=48000$. The detector is a back-illuminated CCD with $2048 \times 4096$ pixels of $15 \mu \mathrm{m}$ size. Reductions were performed using the DRS (online data reduction system of FEROS) and for a subsequent reduction, the TELLURIC, CONTINUUM, RVIDLINES and DOPCOR tasks of the IRAF package were applied.

Precise stellar parameters are fundamental for an acurate inference of the chemical abundances. Chemical abundances are particularly sensitive to the temperature of the stellar atmosphere ( $\left.T_{\text {eff }}\right)$ and the surface gravity of the star $(\log g)$. Two other stellar parameters also play important role in the abundance determination: the metallicity of the star $([\mathrm{Fe} / \mathrm{H}])$ and the microturbulence velocity $(\xi)$. First guesses for the stellar parameters were inferred from photometric data and distances from Hipparcos mission. The final stellar parameters were calculated as follows: temperatures were derived requiring that Fe I lines with different excitation potentials give the same iron abundance; gravities and metallicities were inferred by forcing the agreement between Fe I and Fe II abundances; microturbulence velocities were calculated by requiring no slope in the $[\mathrm{Fe} / \mathrm{H}]$ vs. $E W$ (equivalent width) plot. A detailed description of the entire procedure is given in Pompéia et al. (2008, in preparation). The adopted model atmospheres are an updated version of the planeparallel MARCS model atmospheres with standard composition (Gustafsson et al. 2003). The final parameters and respective uncertainties are given in Table 1.

\section{Stellar abundances}

Abundance analysis has been performed by matching the synthetic profile with the observed spectrum. The line synthesis code is an updated version of the code by Monique Spite (1967) (e.g. Cayrel et al. 2001; Barbuy et al. 2003). The line list and atomic references are given in Table 3. Hyperfine structure (HFS) has been applied for $\mathrm{La}, \mathrm{Ba}, \mathrm{Eu}$ and $\mathrm{Pb}$. The references for the HFS are: Rutten (1978) for Ba II, Lawler et al. (2001a) for La II, Lawler et al. (2001b) for Eu II, and Biémont et al. (2000) for $\mathrm{Pb} \mathrm{I}$.

\subsection{Uncertainties}

Uncertainties on abundances were calculated for both HD 11397 and HD 14282, by verifying how much the variation of $1 \sigma$ of the atmospheric parameters affects the output value of the synthesis program, here $\log A_{\mathrm{p}}$, and also by taking into account the standard deviation of the abundances for which more than 2 lines are available. Table 3 shows the values for this calculation.

Under the simplifying hypothesis of independent errors, the uncertainty of the output value is given by

$\sigma_{\mathrm{Ap}}=\sqrt{\left(\Delta A_{\mathrm{T}}\right)^{2}+\left(\Delta A_{\mathrm{mt}}\right)^{2}+\left(\Delta A_{\mathrm{l}}\right)^{2}+\left(\Delta A_{\xi}\right)^{2}+(s d m)^{2}}$,

where $\Delta A_{\mathrm{T}}, \Delta A_{\mathrm{mt}}, \Delta A_{1}$, and $\Delta A_{\xi}$, are the differences in $A_{\mathrm{p}}$ due to the uncertainties in the temperature, metallicity, $\log g$, and microturbulent velocity respectively and " $s d m$ " is the standard deviation of the average.

The average value of $A_{\mathrm{p}}\left(A_{\mathrm{pm}}\right)$ is obtained by averaging the individual abundances of several lines. Applying a propagation 
Table 2. $\log \epsilon(\mathrm{X})$ and $[\mathrm{X} / \mathrm{Fe}]$ for HD 11397 and HD 14282. The solar abundances used and their sources are shown in Cols. 6 and 7. The numbers in parenthesis are the errors on the last decimals. The estimated stellar parameters for the two stars are: $\mathrm{HD} 11397-T_{\text {eff }}=5400 \mathrm{~K}, \log g=4.15 \mathrm{dex},[\mathrm{Fe} / \mathrm{H}]=-0.72 \mathrm{dex}$ and $\xi=0.80 \mathrm{~km} \mathrm{~s}^{-1}$, HD $14282-T_{\text {eff }}=5800 \mathrm{~K}, \log g=3.70$, $[\mathrm{Fe} / \mathrm{H}]=-0.60 \mathrm{dex}, \xi=1.00 \mathrm{~km} \mathrm{~s}^{-1}$.

\begin{tabular}{ccccccccc}
\hline \hline & \multicolumn{2}{c}{$\mathrm{HD} 11397$} & & \multicolumn{2}{c}{$\mathrm{HD} 14282$} & & \multicolumn{2}{c}{ Sun } \\
\cline { 2 - 3 } el & $\log \epsilon(\mathrm{X})$ & {$[\mathrm{X} / \mathrm{Fe}]$} & & $\log \epsilon(\mathrm{X})$ & {$[\mathrm{X} / \mathrm{Fe}]$} & & $\log \epsilon_{\odot}(\mathrm{X})$ & Ref. \\
\hline $\mathrm{C}$ & $8.14(24)$ & $0.34(20)$ & & $8.28(26)$ & $0.36(22)$ & & $8.52(6)$ & 1 \\
$\mathrm{~N}$ & $7.50(49)$ & $0.30(47)$ & & $7.46(53)$ & $0.14(51)$ & & $7.92(6)$ & 1 \\
$\mathrm{Sr} \mathrm{I}$ & 2.87 & 0.62 & & 3.07 & 0.70 & & $2.97(7)$ & 1 \\
$\mathrm{Sr}$ II & $2.87(26)$ & $0.62(23)$ & & $3.02(25)$ & $0.65(21)$ & & $2.97(7)$ & 1 \\
$\mathrm{Y}$ & $2.17(25)$ & $0.65(20)$ & & $2.21(25)$ & $0.57(21)$ & & $2.24(3)$ & 1 \\
$\mathrm{Zr} \mathrm{I}$ & 2.36 & 0.48 & & 2.55 & 0.55 & & $2.60(2)$ & 1 \\
$\mathrm{Zr} \mathrm{II}$ & $2.59(27)$ & $0.71(23)$ & & $2.53(25)$ & $0.53(20)$ & & $2.60(2)$ & 1 \\
$\mathrm{Mo}$ & $1.52(26)$ & $0.32(22)$ & & $1.72(26)$ & $0.40(22)$ & & $1.92(5)$ & 1 \\
$\mathrm{Ru}$ & $1.96(33)$ & $0.84(31)$ & & $1.54(25)$ & $0.30(21)$ & & $1.84(7)$ & 1 \\
$\mathrm{Ba}$ & $2.36(26)$ & $0.95(21)$ & & $2.04(27)$ & $0.51(23)$ & & $2.13(5)$ & 1 \\
$\mathrm{La}$ & $1.25(26)$ & $0.84(21)$ & & $0.74(25)$ & $0.21(21)$ & & $1.13(3)$ & 2 \\
$\mathrm{Ce}$ & $1.71(25)$ & $0.73(21)$ & & $1.19(25)$ & $0.09(21)$ & & $1.70(4)$ & 3 \\
$\mathrm{Pr}$ & $0.81(38)$ & $0.87(38)$ & & $0.37(32)$ & $0.31(32)$ & & $0.66(15)$ & 4 \\
$\mathrm{Nd}$ & $1.48(25)$ & $0.75(20)$ & & $0.81(25)$ & $-0.04(20)$ & & $1.45(1)$ & 5 \\
$\mathrm{Sm}$ & $0.83(26)$ & $0.54(22)$ & & $0.58(21)$ & $0.17(16)$ & & $1.01(6)$ & 6 \\
$\mathrm{Eu}$ & $0.35(27)$ & $0.55(22)$ & & $0.33(25)$ & $0.41(20)$ & & $0.52(1)$ & 7 \\
$\mathrm{Gd}$ & $0.77(31)$ & $0.37(27)$ & & $0.66(24)$ & $0.14(20)$ & & $1.12(4)$ & 1 \\
$\mathrm{Dy}$ & $0.99(26)$ & $0.51(23)$ & & $0.55(25)$ & $-0.05(21)$ & & $1.20(6)$ & 8 \\
$\mathrm{Hf}$ & $1.11(28)$ & $0.95(25)$ & & $0.58(25)$ & $0.30(22)$ & & $0.88(8)$ & 1 \\
$\mathrm{~Pb}$ & $1.83(45)$ & $0.60(44)$ & & $2.05(41)$ & $0.70(39)$ & & $1.95(8)$ & 1 \\
\hline
\end{tabular}

References on solar abundances: 1 - Grevesse \& Sauval (1998); 2 Lawler et al. (2001a); 3 - Palmeri et al. (2000); 4 - Lage \& Whaling (1976); 5 - Hartog et al. (2003); 6 - Biémont et al. (1989); 7 - Lawler et al. (2001b); 8 - Biémont \& Lowe (1993).

of errors and taking into account the uncertainty calculated with Eq. (1), the uncertainty on $A_{\mathrm{pm}}$ is

$\sigma_{\mathrm{Apm}}=\frac{\sigma_{\mathrm{Ap}}}{\sqrt{n_{1}}}$

where $n_{1}$ is the number of lines for which $\Delta A_{\mathrm{T}}, \Delta A_{\mathrm{mt}}, \Delta A_{1}$, and $\Delta A_{\xi}$ were computed. In the present work $n_{1}=1$, given that we used one line indicated in Table 3 . The uncertainty on the logarithm of $A_{\mathrm{pm}}$ is

$\sigma_{\log (A \mathrm{pm})}=\frac{\sigma_{A \mathrm{pm}}}{A_{\mathrm{pm}} \ln 10}$.

The abundance $\log \epsilon(\mathrm{X})$ is related to the output of the synthesis program by $\log \epsilon(\mathrm{X})=\log A_{\mathrm{pm}}+[\mathrm{Fe} / \mathrm{H}]$. Therefore, the uncertainty is

$\sigma_{\log \epsilon(\mathrm{X})}=\sqrt{\sigma_{\log (A \mathrm{pm})}^{2}+\sigma_{[\mathrm{Fe} / \mathrm{H}]}^{2}}$.

The relation between the abundance excess relative to iron $[\mathrm{X} / \mathrm{Fe}]$ and the output value of the synthesis program is $[\mathrm{X} / \mathrm{Fe}]=\log A_{\mathrm{pm}}-\log \epsilon_{\odot}(\mathrm{X})$, where $\log \epsilon_{\odot}(\mathrm{X})$ is the solar abundance of the element " $\mathrm{X}$ ". The uncertainty is calculated by

$\sigma_{[\mathrm{X} / \mathrm{Fe}]}=\sqrt{\sigma_{\log (\mathrm{Apm})}^{2}+\sigma_{\log \epsilon_{\odot}(\mathrm{X})}^{2}}$.

For $[\mathrm{X} 1 / \mathrm{X} 2]$ the uncertainties are determined by

$\sigma_{[\mathrm{X} 1 / \mathrm{X} 2]}=\sqrt{\sigma_{[\mathrm{X} 1 / \mathrm{Fe}]}^{2}+\sigma_{[\mathrm{X} 2 / \mathrm{Fe}]}^{2}}$,

Uncertainties on elements are shown in parenthesis in Table 2 and by the error bars in figures.
Table 3. Uncertainties on abundances for HD 11397 and HD 14282. " $n$ " is the number of lines used to calculate the abundances; $\lambda$ is the line used to compute the uncertainties; and $\log A_{\mathrm{pf}}$ : output with the atmospheric parameters adopted; $\log A_{\mathrm{pT}}$ : output by altering the adopted $T_{\mathrm{eff}}$ by $100 \mathrm{~K} ; \log A_{\mathrm{pmt}}$ : output by altering +0.15 dex on adopted metallicity; $\log A_{\mathrm{pl}}$ : output by altering +0.2 dex on adopted $\log g \cdot \log A_{\mathrm{p} \xi}$ : output by altering $+0.15 \mathrm{~km} \mathrm{~s}^{-1}$ on adopted microturbulent velocity $\xi ; \log (\mathrm{sdm})$ logarithm of the standard deviation of the average of $A_{\mathrm{p}}$, called $A_{\mathrm{pm}}$. Uncertainties on $\log \epsilon(\mathrm{X})$ and $[\mathrm{X} / \mathrm{Fe}]$ are shown in Table 2.

\begin{tabular}{|c|c|c|c|c|c|c|c|}
\hline \multirow{3}{*}{$\begin{array}{l}\mathrm{el} \\
\mathrm{C}\end{array}$} & \multirow{3}{*}{$\begin{array}{l}n \lambda(\AA) \\
35135.600\end{array}$} & \multicolumn{6}{|c|}{$\log A_{\mathrm{pf}} \log A_{\mathrm{pT}} \log A_{\mathrm{pmt}} \log A_{\mathrm{pl}} \log A_{\mathrm{p} \xi} \log (s d m)$} \\
\hline & & \multicolumn{4}{|c|}{ HD 11397} & \multirow[b]{2}{*}{8.86} & \multirow[b]{2}{*}{$\ldots$} \\
\hline & & 8.86 & 8.91 & 9.01 & 8.86 & & \\
\hline $\mathrm{N}$ & 56478.400 & 8.22 & 8.37 & 8.52 & 8.22 & 8.22 & . \\
\hline $\mathrm{Sr}$ & 24161.79 & 3.59 & 3.62 & 3.76 & 3.64 & 3.57 & 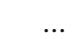 \\
\hline $\mathrm{Y}$ & 125123.21 & 2.86 & 2.91 & 3.01 & 2.91 & 2.81 & 1.37 \\
\hline $\mathrm{Zr}$ & 44317.30 & 3.22 & 3.27 & 3.37 & 3.30 & 3.21 & 2.53 \\
\hline Mo & 15570.439 & 2.24 & 2.34 & 2.39 & 2.24 & 2.24 & 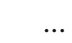 \\
\hline $\mathrm{Ru}$ & 24080.594 & 2.54 & 2.69 & 2.73 & 2.54 & 2.5 & \\
\hline $\mathrm{Ba}$ & 55853.69 & 3.18 & 3.23 & 3.33 & 3.18 & 3.08 & 1.95 \\
\hline $\mathrm{La}$ & 84123.22 & 1.88 & 1.93 & 2.03 & 1.93 & 1.83 & 0.96 \\
\hline $\mathrm{Ce}$ & 104562.36 & 2.45 & 2.50 & 2.60 & 2.50 & 2.4 & 1.20 \\
\hline $\operatorname{Pr}$ & 35220.11 & 1.41 & 1.46 & 1.63 & 1.51 & 1.40 & 0.93 \\
\hline $\mathrm{Nd}$ & 94462.98 & 2.30 & 2.35 & 2.45 & 2.36 & 2.25 & 1.05 \\
\hline $\mathrm{Sm}$ & 54318.93 & 1.36 & 1.41 & 1.51 & 1.41 & 1.35 & 0.66 \\
\hline $\mathrm{Eu}$ & 26645.12 & 1.07 & 1.09 & 1.23 & 1.16 & 1.07 & $\ldots$ \\
\hline $\mathrm{Gd}$ & 34085.57 & 1.42 & 1.47 & 1.62 & 1.47 & 1.42 & 0.51 \\
\hline Dy & 24073.12 & 1.60 & 1.65 & 1.75 & 1.70 & 1.60 & 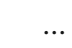 \\
\hline $\mathrm{Hf}$ & 24080.437 & 1.83 & 1.88 & 2.01 & 1.88 & 1.81 & $\cdots$ \\
\hline $\mathrm{Pb}$ & 14057.81 & 2.55 & 2.75 & 2.80 & 2.50 & 2.50 & 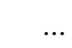 \\
\hline
\end{tabular}

HD 14282

el $n \lambda(\AA) \quad \log A_{\mathrm{pf}} \log A_{\mathrm{pT}} \log A_{\mathrm{pmt}} \log A_{\mathrm{pl}} \log A_{\mathrm{p} \xi} \log (s d m)$

\begin{tabular}{|c|c|c|c|c|c|c|}
\hline $\begin{array}{ll}\mathrm{C} & 45135.600\end{array}$ & 8.85 & 8.94 & 9.00 & 8.83 & 8.85 & $\overline{7.66}$ \\
\hline $\mathrm{N} 36478.400$ & 8.02 & 8.22 & 8.32 & 7.97 & 8.02 & 6.96 \\
\hline Sr $\quad 24161.79$ & 3.67 & 3.72 & 3.82 & 3.72 & 3.62 & \\
\hline Y 115123.21 & 2.89 & 2.94 & 3.04 & 2.94 & 2.84 & 1.81 \\
\hline $\mathrm{Zr} \quad 44317.30$ & 3.10 & 3.15 & 3.25 & 3.16 & 3.09 & 1.91 \\
\hline Mo 15570.439 & 2.32 & 2.42 & 2.47 & 2.37 & 2.32 & \\
\hline Ru $\quad 24080.594$ & 2.14 & 2.20 & 2.29 & 2.19 & 2.14 & \\
\hline Ba 55853.69 & 2.63 & 2.70 & 2.78 & 2.68 & 2.53 & 1.65 \\
\hline La 44123.22 & 1.33 & 1.38 & 1.48 & 1.40 & 1.31 & -0.19 \\
\hline Ce 104562.36 & 1.90 & 1.95 & 2.05 & 1.97 & 1.87 & 0.74 \\
\hline $\operatorname{Pr} \quad 25220.11$ & 1.06 & 1.14 & 1.26 & 1.13 & 1.06 & \\
\hline Nd $\quad 84462.98$ & 1.40 & 1.45 & 1.55 & 1.46 & 1.40 & -0.09 \\
\hline Sm 54318.93 & 1.01 & 1.06 & 1.08 & 1.09 & 1.01 & 0.24 \\
\hline $\mathrm{Eu} \quad 26645.12$ & 0.82 & 0.85 & 0.97 & 0.90 & 0.82 & \\
\hline Gd 34085.57 & 1.22 & 1.25 & 1.37 & 1.27 & 1.22 & 0.16 \\
\hline Dy 24073.12 & 1.10 & 1.15 & 1.25 & 1.17 & 1.10 & \\
\hline Hf 24080.437 & 1.18 & 1.23 & 1.33 & 1.25 & 1.18 & \\
\hline $\mathrm{Pb} \quad 14057.81$ & 2.65 & 2.80 & 2.90 & 2.65 & 2.65 &.. \\
\hline
\end{tabular}

\subsection{Analysis and results}

Abundances for the sample stars are shown in Figs. 1 to 4 . In order to compare our stars with stars of different classifications, we have also added strong and mild Ba-stars to the plots, and normal field stars from Boyarchuk et al. (2002), Liang et al. (2003), Yushchenko et al. (2004), Allen \& Barbuy (2006) and Allen \& Porto de Mello (in preparation).

Carbon and nitrogen: Carbon and $\mathrm{N}$ abundances are plotted in Fig. 1 as a function of $[\mathrm{Fe} / \mathrm{H}]$ and $\log g$. As can be seen 

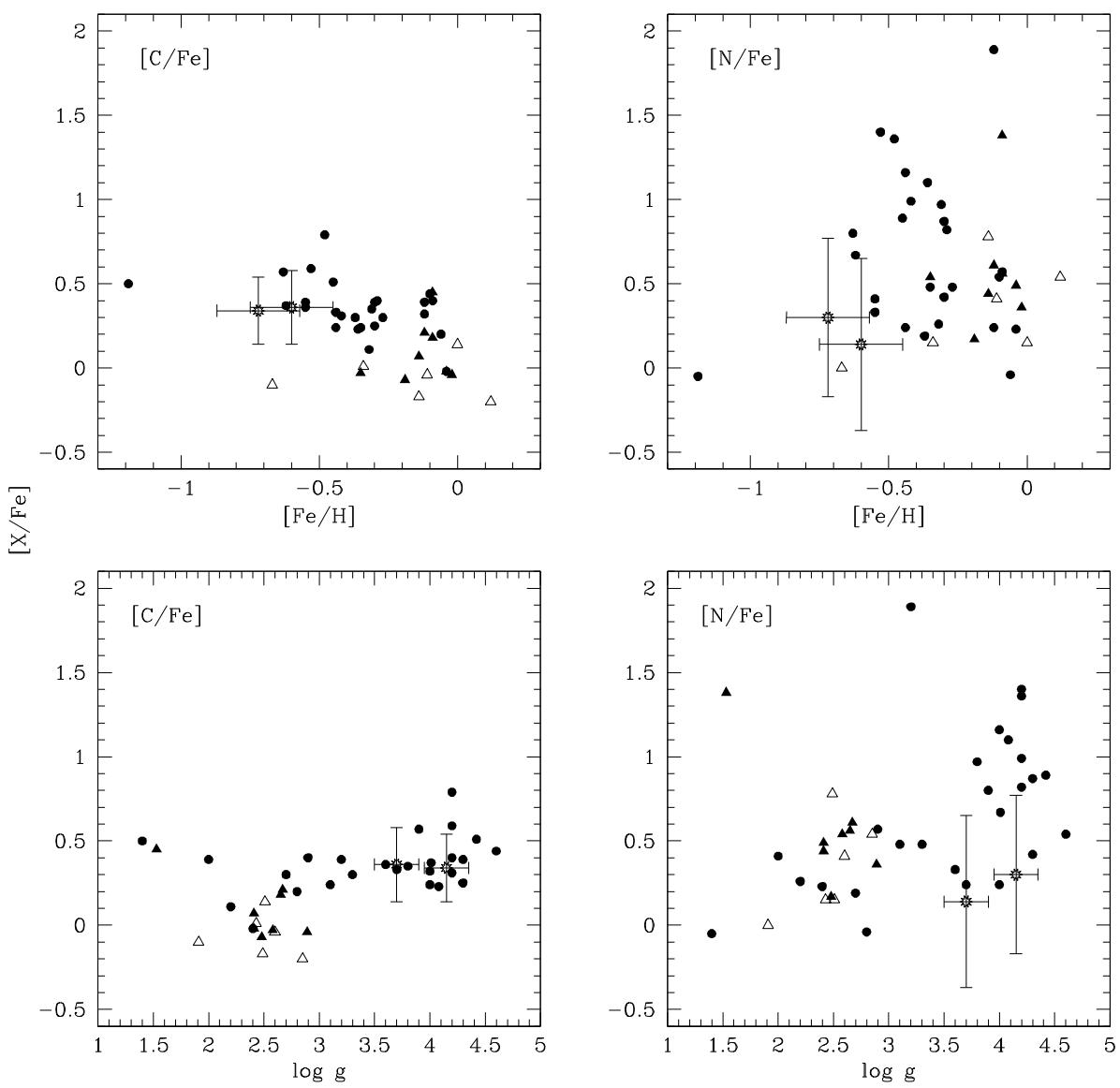

Fig. 1. Upper panels: $[\mathrm{C} / \mathrm{Fe}]$ vs. $[\mathrm{Fe} / \mathrm{H}]$ and $[\mathrm{N} / \mathrm{Fe}]$ vs. $[\mathrm{Fe} / \mathrm{H}]$ for the sample stars. lower panels: $[\mathrm{C} / \mathrm{Fe}]$ and $[\mathrm{N} / \mathrm{Fe}]$ vs. $\log g$. Symbols: starred circles with error bars: this work; filled circles: Allen \& Barbuy (2006); filled triangles: barium stars of Allen \& Porto de Mello (2008); open triangles: stars considered normal rather than barium stars by Allen \& Porto de Mello (2008).

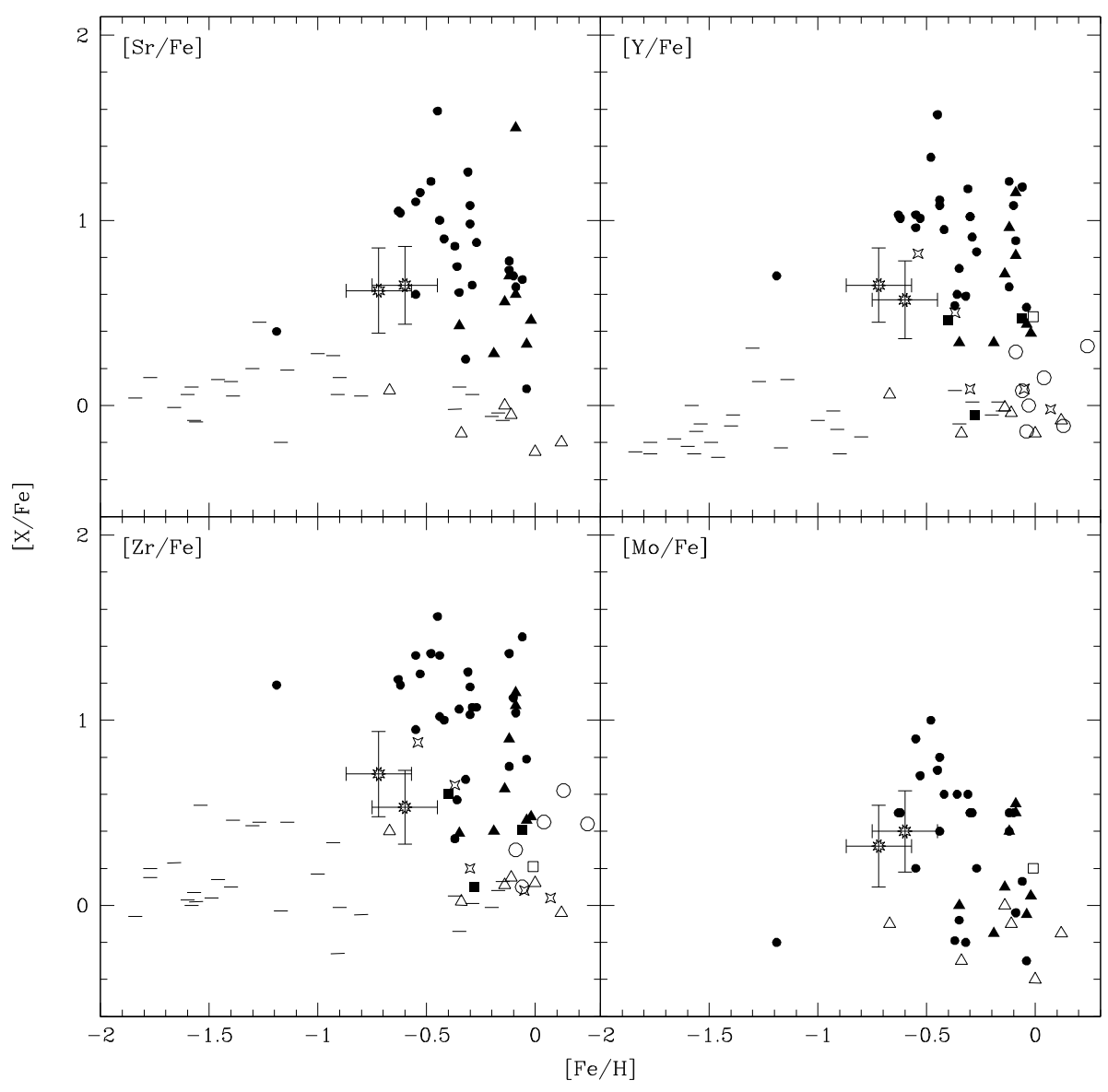

Fig. 2. $[\mathrm{X} / \mathrm{Fe}]$ vs. $[\mathrm{Fe} / \mathrm{H}]$ for the sample stars. Symbols: starred circles with error bars: this work; filled circles: Allen \& Barbuy (2006); filled triangles: barium stars of Allen \& Porto de Mello (2008); open triangles: stars considered normal rather than barium stars by Allen \& Porto de Mello (2008); open circles: stars from Boyarchuk et al. (2002); filled squares: stars from Liang et al. (2003); open squares: stars from Yushchenko et al. (2004); starred squares: barium stars from Edvardsson et al. (1993); dashes: normal stars from Burris et al. (2000), Edvardsson et al. (1993), Gratton \& Sneden (1994), Jehin et al. (1999), Mashonkina \& Gehren (2001), Tomkin \& Lambert (1999), and Woolf et al. (1995). 

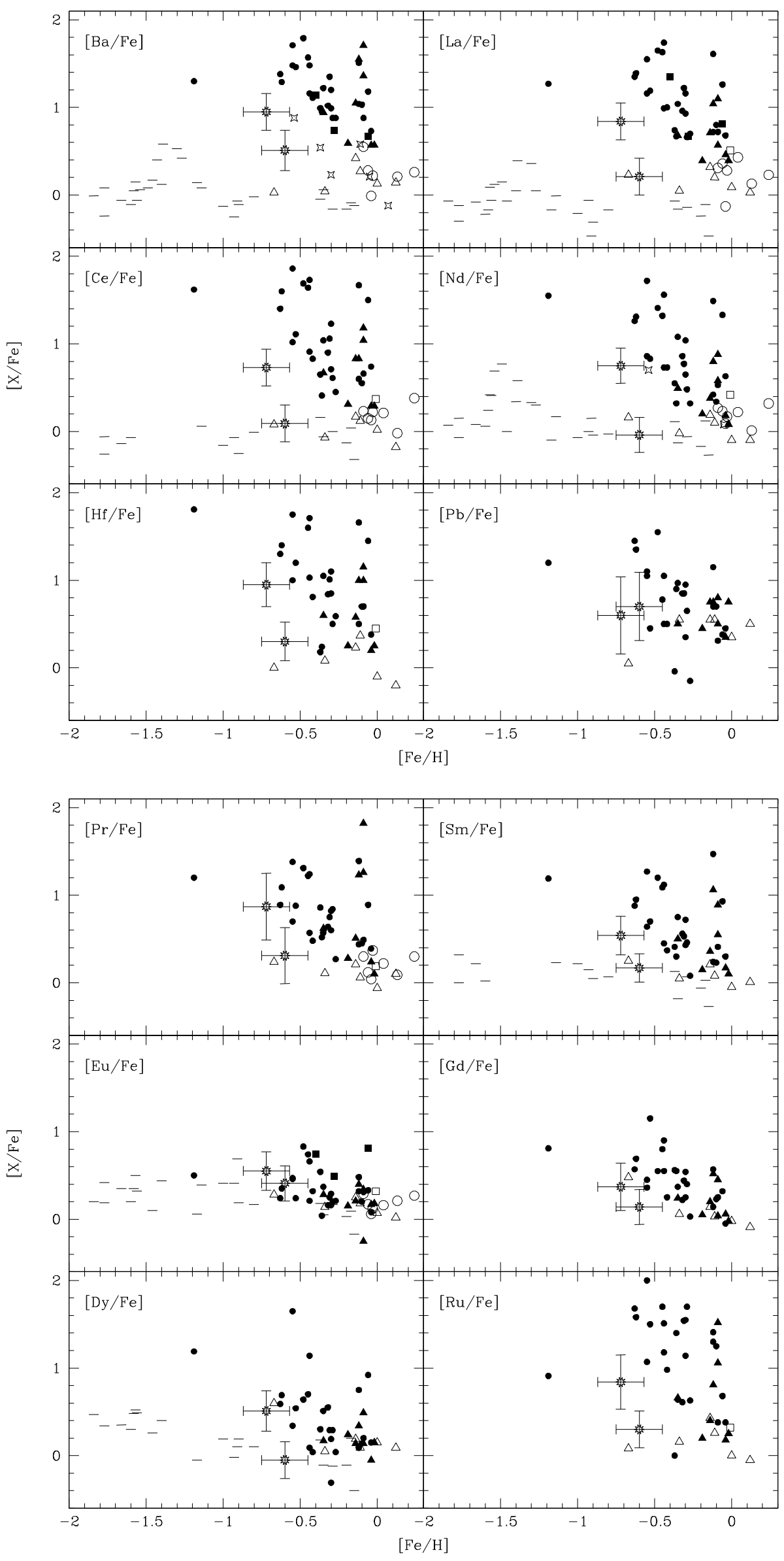

Fig. 3. $[\mathrm{X} / \mathrm{Fe}]$ vs. $[\mathrm{Fe} / \mathrm{H}]$ for the sample stars. Symbols are the same as in Fig. 2.

Fig. 4. $[\mathrm{X} / \mathrm{Fe}]$ vs. $[\mathrm{Fe} / \mathrm{H}]$ for the sample stars. Symbols are the same as in Fig. 2. 
in this figure, $\mathrm{C}$ is slightly enhanced in both HD 11397 and HD 14282 relative to normal stars, matching the Ba stars distributions in both plots, while nitrogen seems normal for the two stars. Compared with the $[\mathrm{C} / \mathrm{H}]$ vs. $[\mathrm{Fe} / \mathrm{H}]$ relation of unpolluted stars from Fig. 11 of Masseron et al. (2006) we also found that both are slightly overabundant, although they can be seemingly considered as normal stars in this plot.

Light s-process elements $-\mathrm{Y}, \mathrm{Zr}, \mathrm{Sr}$ and $\mathrm{Mo}$ : among the light $s$-elements (hereafter $l s$ ) or first $s$-peak elements, $\mathrm{Y}$ is the purest, with a $s$-contribution of $92 \%$ according to Arlandini et al. (1999), followed by $\mathrm{Sr}$ and $\mathrm{Zr}$, with $85 \%$ and $83 \%$, respectively, and $50 \%$ for Mo. In Fig. 2 we can see that, except for $\mathrm{Zr}$, the $l s$ elements in HD 11397 and HD 14282 are mildly high when compared to normal stars, with Y showing the highest value in both stars ( +0.6 dex). HD 14282 shows only slight enhancements for the other $l s$-elements.

Heavy s-process elements - Ba, La, Ce, Nd and Hf: in Figs. 3 and 4 , the distributions for the heavy $s$-process elements (herafter $h s$ ) are depicted. For these elements we have found a clear distinction between the two stars, with HD 11397 showing high $\mathrm{Ba}, \mathrm{Ce}, \mathrm{La}, \mathrm{Hf}$, and $\mathrm{Nd}$ abundances compared to normal stars with similar metallicities. HD 14282 depicts a normal behavior with only a slight enhancement in its $[\mathrm{Ba} / \mathrm{Fe}]$ ratio. $\mathrm{Ba}$ is the $h s$-element or second $s$-peak element (see Busso et al. 1999, 2001) with the highest $s$-contribution $(81 \%)$, followed by $\mathrm{Ce}$ $(77 \%)$ and $\mathrm{La}(62 \%)$ according to Arlandini et al. (1999). Such elements are clearly above the normal stars distribution level in HD 11397.

The termination of the s-process path-Pb: Lead is at the termination of the $s$-process path, or the third peak of the $s$-process. The $s$-contribution for $\mathrm{Pb}$ is still uncertain. Theoretical estimations predict $46 \%$ of yields from the $s$-process (Arlandini et al. 1999), but observations for very low-metallicity stars indicate a strong $s$-contribution, although some contribution from the $r$-process may still be present (e.g. Van Eck et al. 2003; Aoki et al. 2000). Lead seems normal in both HD 11397 and HD 14282.

The mild $s$ and r-elements - Pr, Ru and Sm Praseodymium, $\mathrm{Sm}$ and $\mathrm{Ru}$ have a stronger $r$-contribution, with $51 \%$ for $\operatorname{Pr}, 67 \%$ for $\mathrm{Sm}$, while $\mathrm{Ru}$ has $59 \%$, but they also have an $s$-contribution of $\sim 30 \%$ for $\mathrm{Ru}$ and $\mathrm{Sm}$ and $49 \%$ for Pr. Ru is nearer the first $s$-peak while $\mathrm{Sm}$ and $\mathrm{Pr}$ are nearer the second $s$-peak. These elements also show slight enhancements in HD 11397, while HD 14282 seems to have values similar to those of a normal star.

The r-process dominated elements - Dy, Gd and Eu: Dysprosium, Gd and Eu are dominated by the $r$-process production. In Fig. 4 their distributions are depicted and we see that HD 11397 and HD 14282 show a marginally underabundant behavior for Gd and Dy, while Eu abundances overlap the lower envelope of the Ba-stars and the normal stars distributions, indicating a small $r$-contribution for these stars.

\section{Discussion}

In order to improve our analysis on the abundance profile of the $s$-process elements in HD 11397 and HD 14282, we have compared our data with theoretical surface abundances of AGB stars from Goriely \& Mowlavi (2000) in Fig. 5. If those stars inherited their abundance content from an AGB star, their surface $s$-elements abundances should mimic those of an AGB star. The models are for stars with metallicities similar to our sample stars. Solid lines represent surface abundances after 10 dredge-ups, and dotted lines, after 30 dredge-ups. The upper panel shows

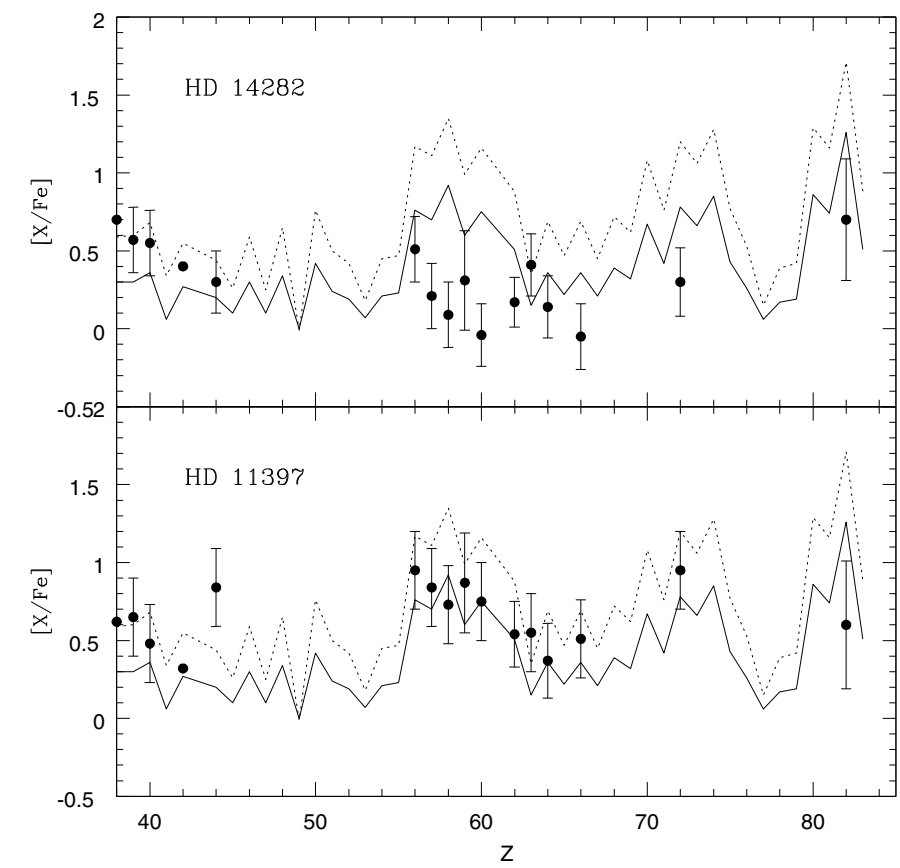

Fig. 5. $[\mathrm{X} / \mathrm{Fe}]$ vs. Z for HD 14282 (upper panel) and HD 11397 (lower panel), compared to theoretical surface abundances predicted for AGB stars of Goriely \& Mowlavi (2000). Our data are compared to models with similar metallicities to the sample stars. Solid lines represent surface abundances predicted for 10 dredge-ups and dotted lines, for 30 dredge-ups.

that most neutron-capture elements in HD 14282 are below the abundances predicted for a 10 dredge-up model. Therefore that star seems to show a slight abundance anomaly for some neutron-capture elements $(\mathrm{Y}, \mathrm{Sr}, \mathrm{Mo}, \mathrm{Pb})$ while other elements are normal. Boyarchuk et al. (2002) have also found some slight $s$-elements anomaly in normal field red giants. If these chemical anomalies in HD 14282 were inherited from the protocloud of the star, such excess should be ascribed to a pristine contamination. If they are due to the beginning of the $s$ process operation and the dredge-up of material enriched in carbon and $s$-elements, HD $14282 s$-anomaly may be ascribed to a mass-transfer mechanism.

The abundances of HD 11397 for most of the elements in Fig. 5 are between the 10 and the 30 dredge-ups curves, in agreement with an AGB abundance profile. Compared to normal disk stars, HD 11397 shows an overabundant behavior for most of the neutron capture elements and seems to share its chemical profile with the mild Ba stars. We suggest that this is also a mild Ba-star. An apparent higher $h s$ content than the $l s$-elements is seen in that star. For both HD 11397 and HD 14282, the heavy element Pb, is underabundant relative to these models predictions.

Radial velocities of the bulgelike stars have been derived using CORAVEL, and possible binaries have been discarded (Grenon 1998). Nevertheless, a detailed study of the radial velocities of HD 11397 and HD 14282 should be performed in order to have a robust statement about their single nature. If their non-binarity is confirmed, they could be used as templates for the study of $s$-enriched stars with a non-binary origin, and therefore in a scenario different from that of the mass-transfer paradigm.

\section{Summary}

We have performed a chemical abundance analysis of two dwarf stars with $s$-process anomalies, HD 11397 and HD 14282. We 
aim to define if they can be considered as Ba-stars, and, if confirmed, to infer their Ba degree, i.e., to define if they are mild or strong Ba stars. Abundances of 18 neutron-capture elements, with different $s$ and/or $r$ processes contributions, have been derived. The resulting abundance ratios of the two stars have been compared to those of normal stars, to abundance ratios of mild and strong Ba stars and to theoretical predictions for AGB stars. We have found that HD 11397 shows a mild enhancement for most of the $s$-process elements as well as for some $r$-process dominated elements. This star seems to share its abundance profile with the mild Ba-stars. Although showing some slight chemical anomalies for Y, Sr and Mo, HD 14282 depicts a chemical pattern similar to the normal stars with slight $s$-process enhancements.

Acknowledgements. Part of this work was supported by the FAPESP fellowship \#01/14594-2. We acknowledge FAPESP project \#98/10138-8. We warmufully thank A. Jorissen for his careful revision of the paper. D.M.A. acknowledges CAPES for the post-doctoral fellowship No. BEX 3448/06-1. We are grateful to Beatriz Barbuy for making available the spectrum synthesis code.

\section{References}

Allen, D. M., \& Barbuy, B. 2006, A\&A, 454, 895

Aoki, W., Norris, J. E., Ryan, S. G., Beers, T. C., \& Ando, H. 2000, ApJ, 536, L97

Arlandini, C., Käppeler, F., Wisshak, K., et al. 1999, ApJ, 525, 886

Barbuy, B., Perrin, M.-N., Katz, D., et al. 2003, A\&A, 404, 661

Beveridge, R. C., \& Sneden, C. 1994, AJ, 108, 285

Bidelman, W. P., \& Keenan, P. C. 1951, ApJ, 114, 473

Biémont, E., \& Lowe, R. M. 1993, A\&A, 273, 665

Biémont, E., Grevesse, N., Hannaford, P., \& Lowe, R. M. 1989, A\&A, 222, 307 Boyarchuk, A. A., Pakhomov, Yu. V., Antipova, L. I., \& Boyarchuk, M. E. 2002, A. Rep., 46, 81

Burris, D. L., Pilachowski, C. A., Armandroff, T. E., et al. 2000, ApJ, 544, 302 Busso, M., Gallino, R., \& Wasserburg, G. J. 1999, ARA\&A, 37, 239

Busso, M., Gallino, R., Lambert, D. L., et al. 2001, ApJ, 557, 802

Cayrel, R., Perrin, M. N., Barbuy, B., \& Buser, R. 1991, A\&A, 247, 108 Edvardsson, B., Andersen, J., Gustafsson, B., et al. 1993, A\&A, 275, 101 Frankowski, A., \& Jorissen, A. 2007, Baltic Astronomy, 16, 104

Goriely, S., \& Mowlavi, N. 2000, 362, 599

Gratton, R. G., \& Sneden, C. 1994, A\&A, 287, 927

Grenon, M. 1998, in Highlights of Astronomy, ed. J. Andersen, 11, 560

Grenon, M. 1999, Ap\&SS, 265, 331
Grenon, M. 2000, in The Evolution of the Milky Way, ed. F. Matteucci, \& F. Giovannelli (Kluwer Academic Publishers), 47

Grevesse, N., \& Sauval, A. J. 1998, Space Sci. Rev., 85, 161

Gustafsson, B., Edvardsson, B., Eriksson, K., et al. 2003, ASP Conf. Ser. 288, ed. I. Hubeny, D. Mihalas, \& K. Werner, 331

Hartog, E. A. D., Lawler, J. E., Sneden, C., \& Cowan, J. J. 2003, ApJS, 148, 543 Iben, I. 1991, ApJS 76, 55

Jehin, E., Magain, P., Neuforge, C., et al. 1999, A\&A, 341, 241

Jorissen, A., \& Van Eck, S. 2005, in Cosmic Abundances as Records of Stellar Evolution and Nucleosynthesis in honor of David L. Lambert, ed. T. G. Barnes III, \& F. N. Bash (San Francisco: ASP), Proc. ASP Conf. Ser., 336, 207

Jorissen, A., Van Eck, S., Mayor, M., \& Udry, S. 1998, A\&A, 332, 877

Karakas, A. I., Tout, C. A., \& Lattanzio, J. C. 2000, MNRAS, 316, 689

Lage, C. S., \& Whaling, W. 1976, JQSRT, 16, 537

Lambert, D. L. 1988, in The Impact of Very High S/N Spectroscopy on Stellar Physics, ed. G. Cayrel de Strobel, \& M. Spite (Dordrecht: Kluwer), IAU Symp., 132, 563

Lambert, D. L., Smith, V. V., \& Heath, J. 1993, PASP, 105, 568

Lawler, J. E., Bonvallet, G., \& Sneden, C. 2001a, ApJ, 556, 452

Lawler, J. E., Wickliffe, M. E., \& Hartog, A. D. 2001b, ApJ, 563, 1075

Liang, Y. C., Zhao, G., \& Zhang, B. 2000, A\&A, 363, 555

Liang, Y. C., Zhao, G., Chen, Y. Q., Qiu, H. M., \& Zhang, B. 2003, A\&A, 397, 257

Luck, R. E., \& Bond, H. E. 1991, ApJS, 77, 515

Malaney, R. A. 1987, Ap\&SS, 137, 251

Mashonkina, L., \& Gehren, T. 2001, A\&A, 376, 232

McClure R. D. 1984, PASP, 96, 117

McClure R. D., \& Woodsworth, A. W. 1990, ApJ, 352, 709

McClure, R. D., Fletcher, J. M., \& Nemec, J. M. 1980, ApJ, 238, L35

Norris, J. E., \& Da Costa, G. S. 1995, ApJ, 447, 680

North, P., Berthet, S., \& Lanz, T. 1994, A\&A, 281, 775

Palmeri, P., Quinet, P., Wyart, J. F., \& Biémont, E. 2000, PhyS, 61, 323

Pols, O. R., Karakas, A. I., Lattanzio, J. C., \& Tout, C. A. 2003, in Symbiotic Stars Probing Stellar Evolution (San Francisco: ASP), ASP Conf. Proc., 303, 290

Pompéia, L., Barbuy, B., \& Grenon, M. 2002, ApJ, 566, 84

Pompéia, L., Barbuy, B., \& Grenon, M. 2003, ApJ, 592, 1173

Rutten, R. J. 1978, SoPhy, 56, 237

Smiljanic, R., Porto de Mello, G. F., \& da Silva, L. 2007, A\&A, 468, 679

Smith, V. V., Suntze, N. B., Cunha, K., et al. 2000, AJ, 119, 1239

Sneden, C., Lambert, D. L., \& Pilachowski, C. A. 1981, ApJ, 247, 1052

Spite, M. 1967, Ann. Astrophys., 30, 211

Tomkin, J., \& Lambert, D. L. 1999, ApJ, 523, 234

Van Eck, S., Goriely, S., Jorissen, A., \& Plez, B. 2003, Nature, 412, 793

Wallerstein, G., Iben, I. Jr., Parker, P., et al. 1997. Rev. Mod. Phys., 69, 995

Warner, B. 1965, MNRAS, 129, 263

Woolf, V. M., Tomkin, J., \& Lambert, D. L. 1995, ApJ, 453, 660

Yushchenko, A. V., Gopka, V. F., Kim, C., et al. 2004, A\&A, 413, 1105 
Table 4. Abundance results for the sample stars. The $G$ band ranges from 4295 to $4315 \AA$.

\begin{tabular}{|c|c|c|c|c|c|c|c|c|c|c|}
\hline \multirow[b]{2}{*}{ el } & \multirow[b]{2}{*}{$\lambda$} & & & \multirow[b]{2}{*}{ Molec } & \multicolumn{3}{|c|}{ HD 11397} & \multicolumn{3}{|c|}{ HD 14282} \\
\hline & & & & & & $\log \epsilon(X)$ & {$[\mathrm{X} / \mathrm{Fe}]$} & & $\log \epsilon(\mathrm{X})$ & {$[\mathrm{X} / \mathrm{Fe}]$} \\
\hline $\mathrm{C}$ & $G$ band & & & $\mathrm{CH}$ & $\ldots$ & 8.14 & 0.34 & $\ldots$ & 8.25 & 0.33 \\
\hline $\mathrm{C}$ & 5135.600 & & & $\mathrm{C} 2$ & $\ldots$ & 8.14 & 0.34 & $\ldots$ & 8.25 & 0.33 \\
\hline $\mathrm{C}$ & 5165.254 & & & C2 $\operatorname{Swan}(0,0)$ & $\ldots$ & 8.14 & 0.34 & $\ldots$ & 8.25 & 0.33 \\
\hline $\mathrm{C}$ & 5635.500 & & & C2 Swan $(0,1)$ & $\ldots$ & $\ldots$ & 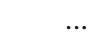 & $\ldots$ & 8.35 & 0.43 \\
\hline $\mathrm{N}$ & 6477.200 & & & $\mathrm{CN}$ & $\ldots$ & 7.50 & 0.30 & $\ldots$ & 7.42 & 0.10 \\
\hline $\mathrm{N}$ & 6478.400 & & & $\mathrm{CN}$ & $\ldots$ & 7.50 & 0.30 & $\ldots$ & 7.42 & 0.10 \\
\hline $\mathrm{N}$ & 6478.700 & & & $\mathrm{CN}$ & $\ldots$ & 7.50 & 0.30 & $\ldots$ & 7.52 & 0.20 \\
\hline $\mathrm{N}$ & 6703.968 & & & $\mathrm{CN}$ & $\ldots$ & 7.50 & 0.30 & $\ldots$ & $\ldots$ & $\ldots$ \\
\hline $\mathrm{N}$ & 6706.733 & & & $\mathrm{CN}$ & $\ldots$ & 7.50 & 0.30 & $\ldots$ & $\ldots$ & $\ldots$ \\
\hline $\mathrm{N}$ & 6708.993 & & & $\mathrm{CN}$ & $\ldots$ & $\ldots$ & $\ldots$ & $\ldots$ & $\ldots$ & $\ldots$ \\
\hline el & $\lambda$ & $\chi_{\mathrm{ex}}$ & $\log g f$ & Ref. & $E W$ & $\log \epsilon(\mathrm{X})$ & {$[\mathrm{X} / \mathrm{Fe}]$} & $E W$ & $\log \epsilon(\mathrm{X})$ & {$[\mathrm{X} / \mathrm{Fe}]$} \\
\hline Sr I & 4607.340 & 0.000 & 0.280 & G94 & 62 & 2.87 & 0.62 & 57 & 3.07 & 0.70 \\
\hline Sr I & 6791.050 & 1.760 & -0.850 & G94 & & & & & & \\
\hline Sr II & 4077.710 & 0.000 & 0.170 & G94 & 274 & 2.87 & 0.62 & 165 & 2.97 & 0.60 \\
\hline Sr II & 4161.790 & 2.940 & -0.600 & G94 & 46 & 2.87 & 0.62 & 49 & 3.07 & 0.70 \\
\hline Y II & 4883.690 & 1.080 & 0.070 & H82 & 75 & 2.24 & 0.72 & 82 & 2.49 & 0.85 \\
\hline Y II & 4982.140 & 1.030 & -1.290 & H82 & 16 & 2.14 & 0.62 & 18 & 2.09 & 0.45 \\
\hline Y II & 5087.430 & 1.080 & -0.170 & H82 & 59 & 2.14 & 0.62 & 67 & 2.19 & 0.55 \\
\hline Y II & 5119.110 & 0.990 & -1.360 & H82 & 34 & 2.19 & 0.67 & 26 & 2.09 & 0.45 \\
\hline Y II & 5123.210 & 0.990 & -0.830 & H82 & 51 & 2.14 & 0.62 & 52 & 2.29 & 0.65 \\
\hline Y II & 5200.420 & 0.990 & -0.570 & H82 & 56 & 2.19 & 0.67 & 57 & 2.19 & 0.55 \\
\hline Y II & 5205.720 & 1.030 & -0.340 & H82 & 59 & 2.09 & 0.57 & 60 & 2.19 & 0.55 \\
\hline Y II & 5289.820 & 1.030 & -1.850 & H82 & 11 & 2.14 & 0.62 & 8 & 2.09 & 0.45 \\
\hline Y II & 5402.780 & 1.840 & -0.520 & HL83 & 23 & 2.14 & 0.62 & 25 & 2.09 & 0.45 \\
\hline Y II & 5473.390 & 1.740 & -1.020 & H82 & 12 & 2.14 & 0.62 & 12 & 2.19 & 0.55 \\
\hline Y II & 5728.890 & 1.840 & -1.120 & H82 & 7 & 2.24 & 0.72 & & & \\
\hline Y II & 6795.410 & 1.730 & -1.250 & M94 & 10 & 2.19 & 0.67 & 22 & 2.19 & 0.55 \\
\hline Zr I & 6127.470 & 0.150 & -1.060 & B81 & 9 & 2.50 & 0.62 & 5 & 2.60 & 0.60 \\
\hline Zr I & 6134.570 & 0.000 & -1.280 & B81 & 7 & 2.30 & 0.42 & $\ldots$ & $\ldots$ & $\cdots$ \\
\hline Zr I & 6140.460 & 0.520 & -1.410 & B81 & 2 & 2.30 & 0.42 & $\ldots$ & $\ldots$ & $\ldots$ \\
\hline Zr I & 6143.180 & 0.070 & -1.100 & B81 & 6 & 2.30 & 0.42 & $\ldots$ & $\ldots$ & $\ldots$ \\
\hline Zr I & 6489.650 & 1.550 & 0.250 & T90, K85 & $\cdots$ & $\cdots$ & $\ldots$ & 3 & 2.50 & 0.50 \\
\hline Zr II & 4208.980 & 0.710 & -0.460 & B81 & 59 & 2.40 & 0.52 & 60 & 2.50 & 0.50 \\
\hline Zr II & 4317.300 & 0.710 & -1.380 & B81 & 32 & 2.50 & 0.62 & 28 & 2.50 & 0.50 \\
\hline Zr II & 5350.090 & 1.830 & -0.930 & T89, K85 & 10 & 2.70 & 0.82 & 11 & 2.50 & 0.50 \\
\hline Zr II & 6114.800 & 1.670 & -1.700 & S00 & 6 & 2.70 & 0.82 & 9 & 2.60 & 0.60 \\
\hline Mo I & 5570.439 & 1.330 & 0.150 & S00 & 13 & 1.52 & 0.32 & 4 & 1.72 & 0.40 \\
\hline Ru I & 4080.594 & 0.810 & -0.040 & VALD & 9 & 1.82 & 0.70 & 2 & 1.54 & 0.30 \\
\hline $\mathrm{Ru} I$ & 4757.856 & 0.928 & -0.540 & ajusol & 8 & 2.07 & 0.95 & 6 & 1.54 & 0.30 \\
\hline Ba II & 4554.030 & 0.000 & 0.170 & M98 & 384 & 2.36 & 0.95 & 198 & 1.93 & 0.40 \\
\hline Ba II & 4934.100 & 0.000 & -0.150 & M98 & 192 & 2.26 & 0.85 & 179 & 1.93 & 0.40 \\
\hline Ba II & 5853.690 & 0.604 & -1.010 & M98 & 119 & 2.46 & 1.05 & 72 & 2.03 & 0.50 \\
\hline Ba II & 6141.727 & 0.704 & -0.070 & M98 & 203 & 2.36 & 0.95 & 120 & 2.13 & 0.60 \\
\hline Ba II & 6496.909 & 0.604 & -0.380 & R78 & 163 & 2.36 & 0.95 & 107 & 2.13 & 0.60 \\
\hline La II & 4086.710 & 0.000 & -0.070 & LS01 & 65 & 1.16 & 0.75 & 43 & 0.73 & 0.20 \\
\hline La II & 4123.220 & 0.320 & 0.130 & LS01 & 69 & 1.16 & 0.75 & 54 & 0.73 & 0.20 \\
\hline La II & 5123.000 & 0.320 & -0.850 & LS01 & 20 & 1.21 & 0.80 & 7 & 0.73 & 0.20 \\
\hline La II & 5303.530 & 0.320 & -1.350 & LS01 & 19 & 1.41 & 1.00 & 7 & 0.78 & 0.25 \\
\hline La II & 5797.570 & 0.240 & -1.360 & LS01 & 10 & 1.26 & 0.85 & $\ldots$ & $\ldots$ & $\ldots$ \\
\hline La II & 5805.770 & 0.120 & -1.560 & LS01 & 13 & 1.16 & 0.75 & $\ldots$ & $\ldots$ & $\ldots$ \\
\hline La II & 5863.710 & 0.930 & -1.370 & LS01 & 4 & 1.41 & 1.00 & $\ldots$ & $\ldots$ & $\ldots$ \\
\hline La II & 6390.492 & 0.320 & -1.410 & LS01 & 11 & 1.16 & 0.75 & $\ldots$ & $\ldots$ & $\ldots$ \\
\hline Ce II & 4073.470 & 0.480 & 0.180 & PQ00 & 31 & 1.63 & 0.65 & 16 & 1.10 & 0.00 \\
\hline Ce II & 4120.830 & 0.320 & -0.290 & PQ00 & 2 & 1.63 & 0.65 & 13 & 1.10 & 0.00 \\
\hline Ce II & 4145.000 & 0.700 & 0.090 & PQ00 & 17 & 1.63 & 0.65 & 8 & 1.10 & 0.00 \\
\hline Ce II & 4222.600 & 0.120 & -0.090 & PQ00 & 38 & 1.63 & 0.65 & 16 & 0.90 & -0.20 \\
\hline Ce II & 4418.780 & 0.860 & 0.230 & PQ00 & 40 & 1.73 & 0.75 & 20 & 1.30 & 0.20 \\
\hline
\end{tabular}


Table 4. continued.

\begin{tabular}{|c|c|c|c|c|c|c|c|c|c|c|}
\hline el & $\bar{\lambda}$ & $\overline{\chi_{\mathrm{ex}}}$ & $\overline{\log g f}$ & Ref. & 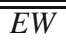 & $\overline{\log \epsilon(\mathrm{X})}$ & $\overline{[\overline{\mathrm{X}} / \mathrm{Fe}]}$ & 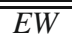 & $\overline{\log \epsilon(\mathrm{X})}$ & $\overline{\overline{[\mathrm{X} / \mathrm{Fe}]}}$ \\
\hline Ce II & 4486.910 & 0.300 & -0.330 & PQ00 & 38 & 1.83 & 0.85 & 17 & 1.30 & 0.20 \\
\hline Ce II & 4523.070 & 0.520 & -0.080 & PQ00 & 34 & 1.63 & 0.65 & 20 & 1.30 & 0.20 \\
\hline Ce II & 4562.360 & 0.480 & 0.190 & PQ00 & 42 & 1.73 & 0.75 & 24 & 1.30 & 0.20 \\
\hline Ce II & 4628.160 & 0.520 & 0.150 & PQ00 & 42 & 1.73 & 0.75 & 21 & 1.10 & 0.00 \\
\hline Ce II & 5330.560 & .870 & -0.510 & PQ00, 1 & 12 & 1.78 & 0.80 & 4 & 1.20 & 0.10 \\
\hline $\mathrm{Ce}$ II & 5975.820 & 1.330 & -0.530 & PQ00 & 10 & 1.83 & 0.85 & & 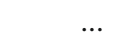 & \\
\hline Pr II & 5220.110 & 0.800 & 0.160 & G91, & 13 & 0.69 & 0.75 & 17 & 0.46 & 0.40 \\
\hline Pr II & 5259.730 & 0.630 & 0.080 & G91, & 11 & 0.69 & 0.75 & 2 & 0.26 & 0.20 \\
\hline Pr II & 5352.410 & 480 & -0.810 & T89, & 5 & 0.99 & 05 & & & \\
\hline Nd II & 4018.820 & 0.060 & -0.850 & $\mathrm{H} 03$ & 24 & 1.38 & .65 & 15 & 0.80 & -0.05 \\
\hline Nd II & 4021.330 & 0.320 & -0.100 & H03 & 36 & 1.43 & 0.70 & 12 & .80 & -0.05 \\
\hline Nd II & 4061.080 & 0.470 & 0.550 & MW7 & 63 & 1.63 & 0.90 & 54 & 0.80 & -0.05 \\
\hline Nd II & 4446.380 & 0.200 & -0.350 & S96 & 30 & 1.38 & 0.65 & 14 & 0.80 & -0.05 \\
\hline Nd II & 4462.980 & 0.560 & 0.040 & MW7 & 40 & 1.58 & 0.85 & 19 & 0.80 & -0.05 \\
\hline Nd II & 5130.590 & 1.300 & 0.450 & H03 & 30 & 1.43 & 0.70 & 12 & 0.80 & -0.05 \\
\hline Nd II & 5319.820 & 0.550 & -0.140 & H03 & 29 & 1.43 & 0.70 & 10 & 0.80 & -0.05 \\
\hline Nd II & 5688.530 & 0.990 & -0.310 & $\mathrm{H} 03$ & 14 & 1.48 & 0.75 & 8 & 0.90 & 0.05 \\
\hline Nd II & 5740.870 & 1.160 & 0.530 & $\mathrm{H} 03$ & 7 & 1.48 & 0.75 & & & \\
\hline Sm II & 4318.930 & 0.280 & 0.270 & B89 & 29 & 0.64 & 0.35 & 30 & 0.41 & 0.00 \\
\hline Sm II & 4499.470 & 250 & 1.010 & B89 & 9 & 0.94 & 0.65 & 5 & 0.61 & 0.20 \\
\hline Sm II & 4537.940 & 0.480 & 0.230 & B89 & 21 & 0.79 & 0.50 & 13 & 0.61 & 0.20 \\
\hline II & 4577.690 & 250 & -0.770 & B89 & 10 & 0.94 & 0.65 & 7 & 0.71 & 0.30 \\
\hline S & 481 & 0.180 & -0.770 & B8 & 11 & 0.74 & 5 & I & 0.51 & 0.10 \\
\hline Eu II & 6437.700 & 1.320 & -0.320 & LD01 & 10 & 0.35 & 0.55 & 10 & 0.42 & 0.50 \\
\hline Eu II & 6645.120 & 1.380 & 0.120 & LD01 & 7 & 0.35 & 0.55 & 9 & 0.22 & 0.30 \\
\hline el & $\lambda$ & $\chi_{\mathrm{ex}}$ & $\overline{\log g f}$ & Ref. & $E W$ & $\epsilon(\mathrm{X})$ & {$[\mathrm{X} / \mathrm{Fe}]$} & $E W$ & $\epsilon(\mathrm{X})$ & $\overline{\mathrm{X} / \mathrm{Fe}]}$ \\
\hline d II & 037.910 & 560 & 0.230 & B8 & 10 & 0.85 & 0. & 4 & 0.62 & 0.10 \\
\hline Gd II & 4085.570 & 730 & 70 & B88 & 6 & 0.70 & 0.3 & 5 & .62 & 0.10 \\
\hline Gd II & 4191.080 & 0.430 & -0.680 & C62 & 10 & 0.75 & 0.3 & 4 & 0.72 & 0.20 \\
\hline Dy II & 4073.120 & 0.540 & -0.330 & K92- & 6 & 0.88 & 0.40 & 5 & 0.50 & -0.10 \\
\hline Dy II & 4103.310 & 0.100 & -0.370 & K92-BI & 29 & 1.08 & 0.6 & 63 & 0.60 & 0.00 \\
\hline Hf II & 4080.437 & 0.608 & -1.050 & aiusol & 15 & 1.11 & 0.95 & 10 & 0.58 & 0.30 \\
\hline Hf II & 4093.155 & 0.452 & -1.090 & VALD & 21 & 1.11 & 0.95 & 5 & 0.58 & 0.30 \\
\hline $\mathrm{Pb} \mathrm{I}$ & 4057.810 & 1.320 & -0.220 & B00 & 111 & 1.83 & 0.60 & 34 & 2.05 & 0.70 \\
\hline
\end{tabular}

$\log \epsilon(\mathrm{X})=\left(\log n_{\mathrm{X}} / n_{\mathrm{H}}\right)+12$ and $[\mathrm{X} / \mathrm{Fe}]=\log \epsilon(\mathrm{X})_{*}-\log \epsilon(\mathrm{X})_{\odot}-[\mathrm{Fe} / \mathrm{H}]$. 